\title{
$q$-Analogues of the sums of consecutive integers, squares, cubes, quarts and quints
}

\author{
Michael Schlosser* \\ Fakultät für Mathematik der Universität Wien \\ Nordbergstraße 15, A-1090 Wien, Austria \\ schlosse@ap.univie.ac.at
}

Submitted: Sep 3, 2004; Accepted: Sep 18, 2004; Published: Sep 30, 2004.

MR Subject Classifications: 33D15, 05A15

\begin{abstract}
We first show how a special case of Jackson's ${ }_{8} \phi_{7}$ summation immediately gives Warnaar's $q$-analogue of the sum of the first $n$ cubes, as well as $q$-analogues of the sums of the first $n$ integers and first $n$ squares. Similarly, by appropriately specializing Bailey's terminating very-well-poised balanced ${ }_{10} \phi_{9}$ transformation and applying the terminating very-well-poised ${ }_{6} \phi_{5}$ summation, we find $q$-analogues for the respective sums of the first $n$ quarts and first $n$ quints. We also derive $q$ analogues of the alternating sums of squares, cubes and quarts, respectively.
\end{abstract}

\section{Introduction}

Garrett and Hummel [2] recently gave a combinatorial proof of a $q$-analogue of the classical formula for the sum of the first $n$ cubes. While the classical formula

$$
\sum_{k=1}^{n} k^{3}=\left(\begin{array}{c}
n+1 \\
2
\end{array}\right)^{2}
$$

is very simple and thus particularly attractive, the $q$-analogue of [2] has the form

$$
\sum_{k=1}^{n} q^{k-1}\left(\frac{1-q^{k}}{1-q}\right)^{2}\left(\frac{1-q^{k-1}}{1-q^{2}}+\frac{1-q^{k+1}}{1-q^{2}}\right)=\left[\begin{array}{c}
n+1 \\
2
\end{array}\right]_{q}^{2} .
$$

Here

$$
\left[\begin{array}{l}
n \\
k
\end{array}\right]_{q}=\prod_{j=1}^{k} \frac{1-q^{n+1-j}}{1-q^{j}}
$$

\footnotetext{
*Supported by Austrian Science Fund FWF, grant P17563-N13
} 
denotes the $q$-binomial coefficient. The identity in (1.2) is called a $q$-analogue of (1.1) since it reduces to the latter by the simple limit $q \rightarrow 1$.

In their paper Garrett and Hummel asked for a simpler $q$-analogue of the sum of cubes (1.1). Further, in view of the fact that there are several classical results for sums of other powers of integers, they suggested it would be natural to develop $q$-analogues of those theorems. Shortly after, as an immediate response to Garrett and Hummel's first question, Warnaar [4] proposed the following identity,

$$
\sum_{k=1}^{n} q^{2 n-2 k} \frac{\left(1-q^{k}\right)^{2}\left(1-q^{2 k}\right)}{(1-q)^{2}\left(1-q^{2}\right)}=\left[\begin{array}{c}
n+1 \\
2
\end{array}\right]_{q}^{2},
$$

which he derived using a simple telescoping argument.

In this paper, we take up on Garrett and Hummel's second question. In particular, we provide $q$-analogues of the classical sums of quarts

$$
\sum_{k=1}^{n} k^{4}=\frac{1}{30} n(n+1)(2 n+1)\left(3 n^{2}+3 n-1\right),
$$

and of quints

$$
\sum_{k=1}^{n} k^{5}=\frac{1}{12} n^{2}(n+1)^{2}\left(2 n^{2}+2 n-1\right),
$$

respectively. We also give some $q$-analogues for alternating sums. We obtain our results (which we deem attractive) by employing specific identities for very-well-poised basic hypergeometric series, in conjunction with suitable specializations of the parameters.

Our paper is organized as follows. In Section 2 we recall some definitions and important identities we need from the theory of basic hypergeometric series. In Section 3 we show how Jackson's very-well-poised balanced ${ }_{8} \phi_{7}$ summation can be directly specialized to recover Warnaar's result (1.4). We also give $q$-analogues of sums of consecutive integers and squares and $q$-analogues of alternating sums of consecutive squares. In Section 4, we suitably specialize Bailey's terminating very-well-poised balanced ${ }_{10} \phi_{9}$ transformation and apply the terminating very-well-poised ${ }_{6} \phi_{5}$ summation on one side of the identity to obtain a little "master identity", see Lemma 1. This identity is then specialized in different ways by which we obtain the desired $q$-analogues of sums of consecutive quarts and quints, and further, $q$-analogues of alternating sums of cubes and quarts. We complete our exposition by speculating about $q$-analogues of sums of higher integer powers.

We would like to thank Martin Rubey for drawing our attention to [4] (which was the starting point of the present paper), and for stimulating discussions.

\section{Preliminaries from basic hypergeometric series}

A standard reference for basic hypergeometric series is Gasper and Rahman's text [3].

Let $q$ be a complex parameter (called the "base"). Since the identities considered in this paper are all terminating (i.e., involve finite sums), we do not require any restriction 
on $q$ (such as $|q|<1$ ). For a complex parameter $a$ and nonnegative integer $k$, the $q$-shifted factorial is defined as

$$
(a ; q)_{k}:=\prod_{j=1}^{k}\left(1-a q^{j-1}\right) .
$$

Empty products are defined to be one, so $(a ; q)_{0}=1$. For brevity of notation, we write

$$
\left(a_{1}, \ldots, a_{m} ; q\right)_{k}=\left(a_{1} ; q\right)_{k} \ldots\left(a_{m} ; q\right)_{k} .
$$

The $q$-binomial coefficient was already defined in (1.3).

The basic hypergeometric ${ }_{r+1} \phi_{r}$ series is defined as

$$
{ }_{r+1} \phi_{r}\left[\begin{array}{c}
a_{1}, a_{2}, \ldots, a_{r+1} \\
b_{1}, b_{2}, \ldots, b_{r}
\end{array} ; q, z\right]:=\sum_{k=0}^{\infty} \frac{\left(a_{1}, \ldots, a_{r+1} ; q\right)_{k}}{\left(q, b_{1}, \ldots, b_{r} ; q\right)_{k}} z^{k} .
$$

The ${ }_{r+1} \phi_{r}$ series terminates if one of the upper parameters, say $a_{r+1}$, is of the form $q^{-n}$ for a nonnegative integer $n$. In this case the series in (2.1) reduces to a sum of $n+1$ terms.

The classical theory of basic hypergeometric series contains several important summation and transformation formulas involving ${ }_{r+1} \phi_{r}$ series. Many of these summation theorems require that the parameters satisfy the condition of being either balanced and/or very-well-poised. An ${ }_{r+1} \phi_{r}$ basic hypergeometric series is called balanced if $b_{1} \cdots b_{r}=$ $a_{1} \cdots a_{r+1} q$ and $z=q$. An ${ }_{r+1} \phi_{r}$ series is well-poised if $a_{1} q=a_{2} b_{1}=\cdots=a_{r+1} b_{r}$ and is very-well-poised if in addition $a_{2}=-a_{3}=q \sqrt{a_{1}}$. Note that this choice of $a_{2}$ and $a_{3}$ entails that the factor

$$
\frac{1-a_{1} q^{2 k}}{1-a_{1}}
$$

appears in a very-well-poised series. The parameter $a_{1}$ is usually referred to as the special parameter of such a series.

We are ready to state some important theorems for very-well-poised series we need in this paper. We start with the most general theorem and then specialize down to simpler identities.

Bailey's transformation formula [3, Appendix (III.28)] for terminating very-well-poised balanced ${ }_{10} \phi_{9}$ series stands on the top of the classical (Bailey) hierarchy of identities for basic hypergeometric series. It contains a number of important transformations and summations as special case. It reads as follows:

$$
\begin{aligned}
& { }_{10} \phi_{9}\left[\begin{array}{c}
a, q \sqrt{a},-q \sqrt{a}, b, c, d, e, f, \lambda a q^{n+1} / e f, q^{-n} \\
\left.\sqrt{a},-\sqrt{a}, a q / b, a q / c, a q / d, a q / e, a q / f, e f q^{-n} / \lambda, a q^{n+1} ; q, q\right]
\end{array}\right] \\
& =\frac{(a q, a q / e f, \lambda q / e, \lambda q / f ; q)_{n}}{(a q / e, a q / f, \lambda q / e f, \lambda q ; q)_{n}} \\
& \times{ }_{10} \phi_{9}\left[\begin{array}{c}
\lambda, q \sqrt{\lambda},-q \sqrt{\lambda}, \lambda b / a, \lambda c / a, \lambda d / a, e, f, \lambda a q^{n+1} / e f, q^{-n} \\
\left.\sqrt{\lambda},-\sqrt{\lambda}, a q / b, a q / c, a q / d, \lambda q / e, \lambda q / f, e f q^{-n} / a, \lambda q^{n+1} ; q, q\right],
\end{array}\right]
\end{aligned}
$$

where $\lambda=q a^{2} / b c d$. 
If in (2.2) we let $d=a q / c$ (thus $\lambda=a / b)$ and suitably relabel parameters $(e \mapsto c$, $f \mapsto d$ ) we obtain Jackson's summation formula [3, Appendix (II.22)] for a terminating very-well-poised balanced ${ }_{8} \phi_{7}$ series.

$$
{ }_{8} \phi_{7}\left[\begin{array}{c}
a, q \sqrt{a},-q \sqrt{a}, b, c, d, a^{2} q^{n+1} / b c d, q^{-n} \\
\sqrt{a},-\sqrt{a}, a q / b, a q / c, a q / d, b c d q^{-n} / a, a q^{n+1} ; q, q
\end{array}\right]=\frac{(a q, a q / b c, a q / b d, a q / c d ; q)_{n}}{(a q / b, a q / c, a q / d, a q / b c d ; q)_{n}} .
$$

Finally, we list the terminating very-well-poised ${ }_{6} \phi_{5}$ summation [3, Appendix (II.22)], obtained from (2.3) by letting $d \rightarrow \infty$.

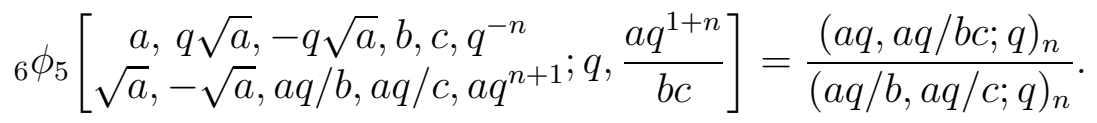

\section{Sums of $q$-integers, $q$-squares and $q$-cubes}

Most likely, Garrett and Hummel initially derived their $q$-analogue of the sum of cubes by assuming the closed form side to be a squared $q$-binomial coefficient, and taking differences. Since

$$
\left[\begin{array}{c}
k+1 \\
2
\end{array}\right]_{q}^{2}-\left[\begin{array}{l}
k \\
2
\end{array}\right]_{q}^{2}=q^{k-1}\left(\frac{1-q^{k}}{1-q}\right)^{2}\left(\frac{1-q^{k-1}}{1-q^{2}}+\frac{1-q^{k+1}}{1-q^{2}}\right),
$$

the identity (1.2) follows immediately by summing both sides of (3.1) over $k$ from 1 to $n$. Warnaar's "achievement" in [4] was to determine that after multiplying the squared $q$-binomial coefficient by a suitable power of $q$, the differences factor nicely into quotients of linear factors.

We show in the following how Jackson's very-well-poised ${ }_{8} \phi_{7}$ summation can be directly specialized to recover Warnaar's result (1.4). Our approach has the advantage that it yields also several other results at the same time. Furthermore, by placing the subject in the appropriate $q$-hypergeometric hierarchy, we are naturally led to the derivation of higher order identities in Section 4.

In (2.3), let $d=a / b c$. This gives the indefinite summation (cf. [3, Eq. (3.6.1)])

$$
\sum_{k=0}^{n} \frac{\left(1-a q^{2 k}\right)}{(1-a)} \frac{(a, b, c, a / b c ; q)_{k}}{(q, a q / b, a q / c, b c q ; q)_{k}} q^{k}=\frac{(a q, b q, c q, a q / b c ; q)_{n}}{(q, a q / b, a q / c, b c q ; q)_{n}} .
$$

Next let $c \rightarrow \infty$. This gives, after multiplying both sides by $b^{n}$,

$$
\sum_{k=0}^{n} \frac{\left(1-a q^{2 k}\right)}{(1-a)} \frac{(a, b ; q)_{k}}{(q, a q / b ; q)_{k}} b^{n-k}=\frac{(a q, b q ; q)_{n}}{(q, a q / b ; q)_{n}} .
$$

To obtain Warnaar's $q$-analogue of the sum of cubes (1.4), replace $n$ by $n-1$, (shift the index of summation, $k \mapsto k-1)$ and set $a=b=q^{2}$.

Of course, our proof of (1.4) is not essentially different from Warnaar's since the indefinite summation (3.2) can also be proved using a telescoping argument. 
Equation (3.3) also contains a $q$-analogue of the sum of the first $n$ squares,

$$
\sum_{k=1}^{n} k^{2}=\frac{1}{6} n(n+1)(2 n+1) .
$$

In (3.3) replace $n$ by $n-1$, then let $a=q^{2}$ and $b=q^{\frac{3}{2}}$, which gives

$$
\sum_{k=1}^{n} \frac{\left(1-q^{2 k}\right)\left(1-q^{k}\right)}{\left(1-q^{2}\right)(1-q)} q^{\frac{3}{2}(n-k)}=\frac{\left(1-q^{n}\right)\left(1-q^{n+1}\right)\left(1-q^{n+\frac{1}{2}}\right)}{(1-q)\left(1-q^{2}\right)\left(1-q^{\frac{3}{2}}\right)} .
$$

Equation (3.3) further contains a $q$-analogue of the sum of the first $n$ integers,

$$
\sum_{k=1}^{n} k=\left(\begin{array}{c}
n+1 \\
2
\end{array}\right)
$$

In (3.3) replace $n$ by $n-1$, then let $a=q^{2}$ and $b=q$, which gives

$$
\sum_{k=1}^{n} \frac{\left(1-q^{2 k}\right)}{\left(1-q^{2}\right)} q^{n-k}=\left[\begin{array}{c}
n+1 \\
2
\end{array}\right]_{q} .
$$

Both identities, (3.4) and (3.5) are different from the corresponding $q$-analogues given by Warnaar in [4], namely

$$
\sum_{k=1}^{n} \frac{\left(1-q^{3 k}\right)\left(1-q^{k}\right)}{\left(1-q^{3}\right)(1-q)} q^{2(n-k)}=\frac{\left(1-q^{n}\right)\left(1-q^{n+1}\right)\left(1-q^{2 n+1}\right)}{(1-q)\left(1-q^{2}\right)\left(1-q^{3}\right)},
$$

and

$$
\sum_{k=1}^{n} \frac{\left(1-q^{k}\right)}{(1-q)} q^{2(n-k)}=\left[\begin{array}{c}
n+1 \\
2
\end{array}\right]_{q}
$$

respectively. These two identities can be easily derived from an indefinite bibasic summation formula due to Gosper (see [3, Eq. (3.6.8)]),

$$
\sum_{k=0}^{n} \frac{\left(1-a p^{k} q^{k}\right)}{(1-a)} \frac{(a ; p)_{k}(c ; q)_{k}}{(q ; q)_{k}(a p / c ; p)_{k}} c^{-k}=\frac{(a p ; p)_{n}(c q ; q)_{n}}{(q ; q)_{n}(a p / c ; p)_{n}} c^{-n}
$$

a formula which is more general than (3.3). First multiply both sides of (3.8) by $c^{n}$, replace $n$ by $n-1$. Then let $c=p=q^{2}$ to obtain (3.6), while let $c=q^{2}$ and $a=0$ to obtain (3.7).

From (3.3) it is also easy to derive a $q$-analogue of the classical formula for the first $n$ odd integers,

$$
\sum_{k=1}^{n}(2 k-1)=n^{2}
$$


In (3.3), simply replace $n$ by $n-1$, and let $a=b=q$, which gives

$$
\sum_{k=1}^{n} \frac{\left(1-q^{2 k-1}\right)}{(1-q)} q^{n-k}=\left(\frac{1-q^{n}}{1-q}\right)^{2}
$$

For a $q$-analogue of the sum of odd squares,

$$
\sum_{k=1}^{n}(2 k-1)^{2}=\frac{1}{3} n(2 n-1)(2 n+1)
$$

replace in (3.3) $n$ by $n-1$, put $a=q$ and $b=q^{\frac{3}{2}}$, which is

$$
\sum_{k=1}^{n} \frac{\left(1-q^{2 k-1}\right)\left(1-q^{k-\frac{1}{2}}\right)}{(1-q)\left(1-q^{\frac{1}{2}}\right)} q^{\frac{3}{2}(n-k)}=\frac{\left(1-q^{n}\right)\left(1-q^{n-\frac{1}{2}}\right)\left(1-q^{n+\frac{1}{2}}\right)}{(1-q)\left(1-q^{\frac{1}{2}}\right)\left(1-q^{\frac{3}{2}}\right)}
$$

We may also obtain from (3.3) results for alternating sums. First replace $n$ by $n-1$, then let $b=-a$. This gives

$$
\sum_{k=1}^{n} \frac{\left(1-a q^{2 k-2}\right)}{(1-a)} \frac{\left(a^{2} ; q^{2}\right)_{k-1}}{\left(q^{2} ; q^{2}\right)_{k-1}}(-1)^{n-k} a^{n-k}=\frac{\left(a^{2} q^{2} ; q^{2}\right)_{n-1}}{\left(q^{2} ; q^{2}\right)_{n-1}} .
$$

Now, for a $q$-analogue of the alternating sum of odd integers,

$$
\sum_{k=1}^{n}(2 k-1)(-1)^{n-k}=n
$$

we simply choose $a=q$ in $(3.11)$ :

$$
\sum_{k=1}^{n} \frac{\left(1-q^{2 k-1}\right)}{(1-q)}(-1)^{n-k} q^{n-k}=\frac{\left(1-q^{2 n}\right)}{\left(1-q^{2}\right)} .
$$

For a $q$-analogue of the alternating sum of squares,

$$
\sum_{k=1}^{n} k^{2}(-1)^{n-k}=\left(\begin{array}{c}
n+1 \\
2
\end{array}\right)
$$

we choose $a=q^{2}$ in (3.11), and then replace $q^{2}$ by $q$ :

$$
\sum_{k=1}^{n}\left(\frac{1-q^{k}}{1-q}\right)^{2}(-1)^{n-k} q^{n-k}=\left[\begin{array}{c}
n+1 \\
2
\end{array}\right]_{q} .
$$

Finally, we point out that the special case $a=q^{2}, b=-q^{\frac{3}{2}}$ of (3.3), with $n$ replaced by $n-1$,

$$
\sum_{k=1}^{n} \frac{\left(1-q^{2 k}\right)\left(1-q^{k}\right)}{\left(1-q^{2}\right)(1-q)}(-1)^{n-k} q^{\frac{3}{2}(n-k)}=\frac{\left(1+q^{n+\frac{1}{2}}\right)}{\left(1+q^{\frac{3}{2}}\right)}\left[\begin{array}{c}
n+1 \\
2
\end{array}\right]_{q},
$$

is another $q$-analogue of (3.13). 


\section{Sums of $q$-quarts and $q$-quints}

In view of the usefulness of the indefinite summation (3.2) obtained from Jackson's summation (2.3), it appears to be indeed appropriate to look for generalizations. By starting with Bailey's transformation formula (2.2), performing some specializations and appealing to a special case of the the terminating ${ }_{6} \phi_{5}$ summation (2.4), we are eventually lead to a little "master identity" in Lemma 1. By specializing this identity in various ways, we obtain $q$-analogues of the sums for consecutive quarts and quints, and $q$-analogues of the alternating sums of cubes and quarts.

In Bailey's transformation formula (2.2), we first let $d=a q^{n+1}$ (thus $\lambda=a q^{-n} / b c$ ), then let $f \rightarrow \infty$, and then put $a=b=q^{2}$. This gives the following transformation of indefinite sums.

$$
\begin{aligned}
& \sum_{k=0}^{n} \frac{\left(1-q^{2+2 k}\right)}{\left(1-q^{2}\right)} \frac{\left(q^{2}, q^{2}, c, e ; q\right)_{k}}{\left(q, q, q^{3} / c, q^{3} / e ; q\right)_{k}}\left(\frac{q}{c e}\right)^{k} \\
& \quad=\frac{\left(q^{3}, c e ; q\right)_{n}}{\left(q^{3} / e, c ; q\right)_{n}} e^{-n} \sum_{k=0}^{n} \frac{\left(1-q^{2 k-n} / c\right)}{\left(1-q^{-n} / c\right)} \frac{\left(q^{-n} / c, q^{-n} / c, q^{-2-n}, e ; q\right)_{k}}{\left(q, q, q^{3} / c, q^{1-n} / c e ; q\right)_{k}}\left(\frac{q^{n+3}}{e}\right)^{k} .
\end{aligned}
$$

Observe now that the sum on the right hand side is almost a terminating very-wellpoised ${ }_{6} \phi_{5}$ series. If the summation index on the right hand side would run from 0 up to $n+2$, we would indeed have a ${ }_{6} \phi_{5}$ series which could be summed using (2.4). However, in the (indefinite) summation above the index runs only up to $n$, two terms too short. Consequently, in order to evaluate the right-hand side of (4.1), we use the simple relation

$$
\sum_{k=0}^{n} a_{k}=\left(\sum_{k=0}^{n+2} a_{k}\right)-a_{n+1}-a_{n+2}
$$

where we can sum the ${ }_{6} \phi_{5}$ series with given summand and then subtract the last two terms. Since by (2.4),

$$
\begin{aligned}
\sum_{k=0}^{n+2} \frac{\left(1-q^{2 k-n} / c\right)}{\left(1-q^{-n} / c\right)} \frac{\left(q^{-n} / c, q^{-n} / c, q^{-2-n}, e ; q\right)_{k}}{\left(q, q, q^{3} / c, q^{1-n} / c e ; q\right)_{k}}\left(\frac{q^{n+3}}{e}\right)^{k} & =\frac{\left(q^{1-n} / c, q / e ; q\right)_{n+2}}{\left(q, q^{1-n} / c e ; q\right)_{n+2}} \\
& =\frac{\left(c / q^{2}, q / e ; q\right)_{n+2}}{\left(q, c e / q^{2} ; q\right)_{n+2}} e^{n+2}
\end{aligned}
$$

we have

$$
\sum_{k=0}^{n} \frac{\left(1-q^{2 k-n} / c\right)}{\left(1-q^{-n} / c\right)} \frac{\left(q^{-n} / c, q^{-n} / c, q^{-2-n}, e ; q\right)_{k}}{\left(q, q, q^{3} / c, q^{1-n} / c e ; q\right)_{k}}\left(\frac{q^{n+3}}{e}\right)^{k}
$$




$$
\begin{aligned}
&=\frac{\left(c / q^{2}, q / e ; q\right)_{n+2}}{\left(q, c e / q^{2} ; q\right)_{n+2}} e^{n+2}- \frac{\left(1-q^{n+2} / c\right)}{\left(1-q^{-n} / c\right)} \frac{\left(q^{-n} / c, q^{-n} / c, q^{-2-n}, e ; q\right)_{n+1}}{\left(q, q, q^{3} / c, q^{1-n} / c e ; q\right)_{n+1}}\left(\frac{q^{n+3}}{e}\right)^{n+1} \\
&- \frac{\left(1-q^{n+4} / c\right)}{\left(1-q^{-n} / c\right)} \frac{\left(q^{-n} / c, q^{-n} / c, q^{-2-n}, e ; q\right)_{n+2}}{\left(q, q, q^{3} / c, q^{1-n} / c e ; q\right)_{n+2}}\left(\frac{q^{n+3}}{e}\right)^{n+2} \\
&=\frac{(c / q ; q)_{n+1}(q / e ; q)_{n+2}}{(c e / q ; q)_{n+1}(q ; q)_{n+2}} {\left[\frac{\left(1-c / q^{2}\right)}{\left(1-c e / q^{2}\right)} e^{n+2}+\frac{(e ; q)_{n+1}(c / q ; q)_{n+2}}{\left(q^{3} / c ; q\right)_{n+1}(q / e ; q)_{n+2}}\left(\frac{q}{c}\right)^{n}\right.} \\
&\left.\times\left(\frac{\left(1-q^{n+2} / c\right)\left(1-q^{n+2}\right)^{2}}{(1-q)(1-c / q)^{2}}+\frac{\left(1-e q^{n+1}\right)}{\left(1-c e / q^{2}\right)} \frac{q^{n+2}}{c}\right)\right] .
\end{aligned}
$$

In the last equality we have applied some elementary manipulations for $q$-shifted factorials (see e.g. [3, Appendix I]) which allowed us to simplify the result significantly. We now substitute the last expression for the sum on the right hand side of (4.1) and obtain

$$
\begin{aligned}
& \sum_{k=0}^{n} \frac{\left(1-q^{2+2 k}\right)}{\left(1-q^{2}\right)} \frac{\left(q^{2}, q^{2}, c, e ; q\right)_{k}}{\left(q, q, q^{3} / c, q^{3} / e ; q\right)_{k}}\left(\frac{q}{c e}\right)^{k} \\
&=\frac{(1-c / q)(1-q / e)\left(1-q^{2} / e\right)}{(1-c e / q)(1-q)\left(1-q^{2}\right)} e^{-n} {\left[\frac{\left(1-c / q^{2}\right)}{\left(1-c e / q^{2}\right)} e^{n+2}+\frac{(e ; q)_{n+1}(c / q ; q)_{n+2}}{\left(q^{3} / c ; q\right)_{n+1}(q / e ; q)_{n+2}}\left(\frac{q}{c}\right)^{n}\right.} \\
&\left.\times\left(\frac{\left(1-q^{n+2} / c\right)\left(1-q^{n+2}\right)^{2}}{(1-q)(1-c / q)^{2}}+\frac{\left(1-e q^{n+1}\right)}{\left(1-c e / q^{2}\right)} \frac{q^{n+2}}{c}\right)\right] \\
&=\frac{(1-c / q)(1-q / e)\left(1-q^{2} / e\right)}{(1-c e / q)(1-q)\left(1-q^{2}\right)} e^{-n} {\left[\frac{\left(1-c / q^{2}\right)}{\left(1-c e / q^{2}\right)} e^{n+2}+\frac{(e ; q)_{n+1}(c ; q)_{n+1}}{\left(q^{3} / c ; q\right)_{n}(q / e ; q)_{n+2}}\left(\frac{q}{c}\right)^{n}\right.} \\
&\left.\times\left(\frac{\left(1-q^{n+1}\right)\left(1-q^{n+2}\right)}{(1-q)(1-c / q)}-\frac{(1-e / q)}{\left(1-c e / q^{2}\right)} q^{n+1}\right)\right] .
\end{aligned}
$$

In the second equality we have pulled out the quotient of linear factors $\left(1-q^{n+3} / c\right) /(1-$ $c / q$ ) from the sum of the two terms inside the big parentheses. This will be convenient for our purpose, in particular for deriving nice $q$-analogues of the formulas (1.5) and (1.6). The products outside the big parentheses will contain the linear factors appearing in the respective formulas while the expression inside the big parentheses (which is a sum of two terms) contributes a nonlinear, quadratic factor.

We manipulate the identity in (4.2) slightly further. After replacing $n$ by $n-1$, shifting the index of summation $(k \mapsto k-1)$, and multiplying both sides by $(c e / q)^{n-1}$ we arrive at the following result.

\section{Lemma 1}

$$
\sum_{k=1}^{n} \frac{\left(1-q^{2 k}\right)}{\left(1-q^{2}\right)}\left(\frac{1-q^{k}}{1-q}\right)^{2} \frac{(c, e ; q)_{k-1}}{\left(q^{3} / c, q^{3} / e ; q\right)_{k-1}}\left(\frac{c e}{q}\right)^{n-k}
$$




$$
\begin{aligned}
=\frac{(1-c / q)(1-q / e)\left(1-q^{2} / e\right)}{(1-c e / q)(1-q)\left(1-q^{2}\right)} & {\left[\frac{\left(1-c / q^{2}\right)}{\left(1-c e / q^{2}\right)} e^{2}\left(\frac{c e}{q}\right)^{n-1}+\frac{(e ; q)_{n}(c ; q)_{n}}{\left(q^{3} / c ; q\right)_{n-1}(q / e ; q)_{n+1}}\right.} \\
& \left.\times\left(\frac{\left(1-q^{n}\right)\left(1-q^{n+1}\right)}{(1-q)(1-c / q)}-\frac{(1-e / q)}{\left(1-c e / q^{2}\right)} q^{n}\right)\right] .
\end{aligned}
$$

When $c=q^{2}$, the right hand side of (4.3) simplifies considerably since one term drops out. We find it convenient to list this special case explicitly.

\section{Corollary 2}

$$
\begin{aligned}
& \sum_{k=1}^{n} \frac{\left(1-q^{2 k}\right)}{\left(1-q^{2}\right)}\left(\frac{1-q^{k}}{1-q}\right)^{3} \frac{(e ; q)_{k-1}}{\left(q^{3} / e ; q\right)_{k-1}}(e q)^{n-k} \\
& \quad=\frac{\left(1-q^{n}\right)\left(1-q^{n+1}\right)(e ; q)_{n}}{(1-q)\left(1-q^{2}\right)(1-e q)\left(q^{3} / e ; q\right)_{n-1}}\left(\frac{\left(1-q^{n}\right)\left(1-q^{n+1}\right)}{(1-q)^{2}}-\frac{(1-e / q)}{(1-e)} q^{n}\right) .
\end{aligned}
$$

The case $e=q^{\frac{3}{2}}$ of Lemma 1 will also be needed.

\section{Corollary 3}

$$
\begin{aligned}
\sum_{k=1}^{n} & \frac{\left(1-q^{2 k}\right)}{\left(1-q^{2}\right)}\left(\frac{1-q^{k}}{1-q}\right)^{2} \frac{(c ; q)_{k-1}}{\left(q^{3} / c ; q\right)_{k-1}}\left(c q^{\frac{1}{2}}\right)^{(n-k)} \\
= & \frac{(1-c / q)\left(1-q^{-\frac{1}{2}}\right)\left(1-q^{\frac{1}{2}}\right)}{\left(1-c q^{\frac{1}{2}}\right)(1-q)\left(1-q^{2}\right)}\left[\frac{\left(1-c / q^{2}\right)}{\left(1-c q^{-\frac{1}{2}}\right)} q^{3}\left(c q^{\frac{1}{2}}\right)^{n-1}\right. \\
& \left.\quad+\frac{\left(1-q^{n+\frac{1}{2}}\right)(c ; q)_{n}}{\left(1-q^{-\frac{1}{2}}\right)\left(1-q^{\frac{1}{2}}\right)\left(q^{3} / c ; q\right)_{n-1}}\left(\frac{\left(1-q^{n}\right)\left(1-q^{n+1}\right)}{(1-q)(1-c / q)}-\frac{\left(1-q^{\frac{1}{2}}\right)}{\left(1-c q^{-\frac{1}{2}}\right)} q^{n}\right)\right] .
\end{aligned}
$$

In the sequel, we consider special cases of Corollaries 2 and 3 leading to $q$-analogues of several sums and alternating sums of low integer powers.

The first thing we observe is that by letting $e=q$ in Corollary 2 we immediately recover Warnaar's q-analogue of the sum of cubes in (1.4). (One could in principal obtain the same result with putting $e=q^{3} / c$ directly in Lemma 1 , however it is not easy to see at one glance that the right-hand side then is independent of $c$ and factors correctly to the squared $q$-binomial coefficient as desired.)

Next, we take $e=q^{\frac{3}{2}}$ in Corollary 2. This gives

$$
\begin{aligned}
& \sum_{k=1}^{n} \frac{\left(1-q^{2 k}\right)}{\left(1-q^{2}\right)}\left(\frac{1-q^{k}}{1-q}\right)^{3} q^{\frac{5}{2}(n-k)} \\
& \quad=\frac{\left(1-q^{n}\right)\left(1-q^{n+1}\right)\left(1-q^{n+\frac{1}{2}}\right)}{(1-q)\left(1-q^{2}\right)\left(1-q^{\frac{5}{2}}\right)}\left(\frac{\left(1-q^{n}\right)\left(1-q^{n+1}\right)}{(1-q)^{2}}-\frac{\left(1-q^{\frac{1}{2}}\right)}{\left(1-q^{\frac{3}{2}}\right)} q^{n}\right) .
\end{aligned}
$$


This is clearly a $q$-analogue of the classical sum of quarts (1.5), as $3 n^{2}+3 n-1=$ $3 n(n+1)-1$.

The special case $e=q^{2}$ of Corollary 2 gives

$$
\begin{aligned}
\sum_{k=1}^{n} \frac{\left(1-q^{2 k}\right)}{\left(1-q^{2}\right)}\left(\frac{1-q^{k}}{1-q}\right)^{4} q^{3(n-k)} & \\
& =\frac{\left(1-q^{n}\right)^{2}\left(1-q^{n+1}\right)^{2}}{(1-q)^{2}\left(1-q^{2}\right)\left(1-q^{3}\right)}\left(\frac{\left(1-q^{n}\right)\left(1-q^{n+1}\right)}{(1-q)^{2}}-\frac{(1-q)}{\left(1-q^{2}\right)} q^{n}\right) .
\end{aligned}
$$

This is clearly a $q$-analogue of the classical sum of quints (1.6), as $2 n^{2}+2 n-1=$ $2 n(n+1)-1$.

The special cases of Corollary 2 where $e=-q^{\frac{3}{2}}, e=-q^{2}, e \rightarrow 0$ and $e \rightarrow \infty$ (in the latter two cases we first need to multiply both sides of (4.4) by $e^{1-n}$ ) all give $q$-analogues of the alternating sum of quarts, which in the classical case reads as

$$
\sum_{k=1}^{n} k^{4}(-1)^{n-k}=\frac{1}{2} n(n+1)\left(n^{2}+n-1\right) .
$$

In particular, we list the two cases $e=-q^{\frac{3}{2}}$ and $e=-q^{2}$ of Corollary 2 explicitly:

$$
\begin{aligned}
\sum_{k=1}^{n} \frac{\left(1-q^{2 k}\right)}{\left(1-q^{2}\right)} & \left(\frac{1-q^{k}}{1-q}\right)^{3}(-1)^{n-k} q^{\frac{5}{2}(n-k)} \\
= & \frac{\left(1-q^{n}\right)\left(1-q^{n+1}\right)\left(1+q^{n+\frac{1}{2}}\right)}{(1-q)\left(1-q^{2}\right)\left(1+q^{\frac{5}{2}}\right)}\left(\frac{\left(1-q^{n}\right)\left(1-q^{n+1}\right)}{(1-q)^{2}}-\frac{\left(1+q^{\frac{1}{2}}\right)}{\left(1+q^{\frac{3}{2}}\right)} q^{n}\right)
\end{aligned}
$$

and

$$
\begin{aligned}
\sum_{k=1}^{n}\left(\frac{1-q^{2 k}}{1-q^{2}}\right)^{2} & \left(\frac{1-q^{k}}{1-q}\right)^{2}(-1)^{n-k} q^{3(n-k)} \\
& =\frac{\left(1-q^{2 n}\right)\left(1-q^{2(n+1)}\right)}{(1-q)\left(1-q^{2}\right)^{2}\left(1+q^{3}\right)}\left(\frac{\left(1-q^{n}\right)\left(1-q^{n+1}\right)}{(1-q)^{2}}-\frac{(1+q)}{\left(1+q^{2}\right)} q^{n}\right),
\end{aligned}
$$

both of which are clearly $q$-analogues of $(4.8)$, as $n^{2}+n-1=n(n+1)-1$.

Finally, we give $q$-analogues of the alternating sum of cubes, which in the classical case reads as

$$
\sum_{k=1}^{n} k^{3}(-1)^{n-k}=\frac{1}{8}\left[(2 n+1)\left(2 n^{2}+2 n-1\right)+(-1)^{n}\right] .
$$


Take $c=-q^{\frac{3}{2}}$ in Corollary 3. This gives

$$
\begin{aligned}
\sum_{k=1}^{n} \frac{\left(1-q^{2 k}\right)}{\left(1-q^{2}\right)}\left(\frac{1-q^{k}}{1-q}\right)^{2}(-1)^{n-k} q^{2(n-k)} & \\
=\frac{\left(1-q^{-\frac{1}{2}}\right)}{\left(1-q^{4}\right)}\left[\frac{\left(1+q^{-\frac{1}{2}}\right)}{(1+q)} q^{2(n+1)}(-1)^{n-1}\right. & \\
& \left.+\frac{\left(1-q^{2 n+1}\right)}{\left(1-q^{-\frac{1}{2}}\right)\left(1-q^{\frac{1}{2}}\right)}\left(\frac{\left(1-q^{n}\right)\left(1-q^{n+1}\right)}{(1-q)\left(1+q^{\frac{1}{2}}\right)}-\frac{\left(1-q^{\frac{1}{2}}\right)}{(1+q)} q^{n}\right)\right],
\end{aligned}
$$

which is a $q$-analogue of (4.11). The $c=-q^{2}$ case of Corollary 3 gives another $q$-analogue of (4.11):

$$
\begin{aligned}
\sum_{k=1}^{n}\left(\frac{1-q^{2 k}}{1-q^{2}}\right)^{2}\left(\frac{1-q^{k}}{1-q}\right)(-1)^{n-k} q^{\frac{5}{2}(n-k)} & \\
= & \frac{(1+q)\left(1-q^{-\frac{1}{2}}\right)\left(1-q^{\frac{1}{2}}\right)}{\left(1+q^{\frac{5}{2}}\right)(1-q)\left(1-q^{2}\right)}\left[\frac{2}{\left(1+q^{\frac{3}{2}}\right)} q^{3}(-1)^{n-1}\left(q^{\frac{5}{2}}\right)^{n-1}\right. \\
& \left.\quad+\frac{\left(1-q^{n+\frac{1}{2}}\right)\left(1+q^{n-1}\right)\left(1+q^{n}\right)}{\left(1-q^{-\frac{1}{2}}\right)\left(1-q^{\frac{1}{2}}\right)(1+q)}\left(\frac{\left(1-q^{n}\right)\left(1-q^{n+1}\right)}{(1-q)(1+q)}-\frac{\left(1-q^{\frac{1}{2}}\right)}{\left(1+q^{\frac{3}{2}}\right)} q^{n}\right)\right] .
\end{aligned}
$$

One can derive two other $q$-analogues of (4.11) by multiplying both sides of (4.5) by $c^{1-n}$ and taking the limit $c \rightarrow 0$, or $c \rightarrow \infty$.

Concluding Remark 4 Our $q$-analogues of $\sum_{k=1}^{n} k^{m}$, for $m=1,2,3,4,5$, all are of the form

$$
\sum_{k=1}^{n} \frac{\left(1-q^{2 k}\right)}{\left(1-q^{2}\right)}\left(\frac{1-q^{k}}{1-q}\right)^{m-1} q^{\frac{m+1}{2}(n-k)},
$$

compare with (3.5), (3.4), (1.4), (4.6) and (4.7), respectively. It is a good guess that a reasonable continuation involving higher integer powers will follow the same pattern. It is not clear whether, for instance, by appropriately specializing Sears' general (multi-term) transformation for well-poised basic hypergeometric series (cf. [3, Sec. 4.12]), one can derive general formulas. These may explicitly involve $q$-Bernoulli numbers or $q$-Bernoulli polynomials (which could be but would not need to be those defined by Carlitz [1]). On the other hand, a general formula for (4.14) may even eventually lead to a solid definition for (a possibly new type of) $q$-Bernoulli polynomials. This should be worth investigating. In any case, it should be at least possible to successively determine closed forms for (4.14) explicitly for small values of $m$ greater than 5 , say for $m=6,7,8,9$.

\section{References}

[1] L. Carlitz, "q-Bernoulli numbers and polynomials", Duke Math. J. 15 (4) (1948), $987-1000$. 
[2] K. C. Garrett and K. Hummel, "A combinatorial proof of the sum of $q$-cubes", Electron. J. Comb. 11 (2004), \#R9.

[3] G. Gasper and M. Rahman, Basic hypergeometric series, Encyclopedia of Mathematics And Its Applications 35, Cambridge University Press, Cambridge, 1990.

[4] S. O. Warnaar, "On the $q$-analogue of the sum of cubes", Electron. J. Comb. 11 (2004), \#N13. 